\title{
Relationship between occupational physical activity and subclinical vascular damage in high-altitude
} dwellers

\begin{abstract}
Abstact
Introduction: Occupational physical activity (PA) has been associated with increased cardiovascular (CV) events. The aim of this study is to investigate the association between occupational PA and markers of subclinical vascular damage among a moderate altitude population living in the rural village of Chauricharka (Nepal 2600m s.l.)

Methods: 72 individuals (age 42 \pm 15 yrs, age range 15-85, 23 men) were enrolled. PA was evaluated by international physical activity questionnaire (IPAQ). Carotid-femoral pulse wave velocity (PWV), carotid ultrasound assessment of geometry and stiffness, flow mediated dilation (FMD) and glyceril trinitrate mediated dilation (GTN) were performed. Medical history, brachial blood pressure, and anthropometric parameters were taken. A blood sample was taken to study metabolic profile, renal function and hemoglobin. Framingham risk score (FRS) was calculated
\end{abstract}

Results: In subjects enrolled the prevalence of hypertension was 33\%, hypercholesterolemia 44\% (LDL $>130 \mathrm{mg} / \mathrm{dl}$ ) and the FRS was $1 \%$. Oxygen saturation and mean hemoglobin were in the normal range for high altitude dwellers $(94.1 \pm 1.6 \%$ and $15.0 \pm 1.3 \mathrm{mg} / \mathrm{dl}$ respectively). PWV, carotid IMT, FMD and GTN were normal. Occupational PA was $9860 \pm 5385$ MET-minutes/week representing $77 \%$ of total energy expenditure with $97 \%$ of the population performing a high level of PA. At the univariate analysis occupational PA was significantly associated with PWV $(\mathrm{R}=0.474$, $\mathrm{p}=0.001)$ and $\mathrm{CS}(\mathrm{R}=0.29, \mathrm{p}=0.019)$. At the multivariate analysis, including age, sex, $\mathrm{SO}_{2}$, mean $\mathrm{BP}$, oxygen saturation and LDL, occupational PA remained an independent predictor of PWV ( $\beta=0.403, p=0.001)$ but not of $C S(R=0.028, p=0.8)$. From multiple regression analysis the association of occupational PA and FRS $(\mathrm{R}=0.672, \mathrm{p}<0.0001)$ were found to be better predictors of PWV than FRS alone $(\mathrm{R}=0.24, \mathrm{p}=0.039)$.

Conclusion: High-intensity occupational PA shows a positive, independent association with arterial stiffness in Himalayan moderate altitude dwellers. This study suggests how occupational PA may be a new CV risk factor and thus should be included in the assessment of overall CV risk

Key words: occupational physical activity, arterial stiffness, atherosclerosis, cardiovascular disease, cardiovascular risk. 


\section{Introduction}

Cardiovascular (CV) diseases are the leading cause of mortality in the world with approximately 235 deaths per 100.000 every year [1]. To reduce CV mortality and morbility many preventive strategies have been suggested [2-5]. Physical activity (PA) is a well-known that improves the overall health and reduces the risk of CV events [5-8] whereas a sedentary lifestyle is associated with an increased risk of CV diseases [9, 10]. On this basis the US department of Health and Human Services, in the last report, recommends a minimum of 800 MET-minutes per week of dynamic aerobic PA as a standard strategy for reducing CV mortality and morbidity [11].

However, while most of the scientific literature has focused the attention on leisure time PA and its benefits in improving CV health, less is known regarding the association between occupational PA and CV risks or benefits. While few studies have found an association between high-levels of workrelated PA and a reduced risk of CV diseases [12,13], others have found an inverse correlation or no correlation at all [14-20]. These inconclusive findings are mainly due to the fact that most of the studies don't consider separately occupational from leisure time PA. Moreover, little is known regarding potential effect of ethnicity, especially adaptation to altitude so further studies are needed. I think we should take into account and briefly introduce the following paper: Lewis NC et al. Conduit artery structure and function in lowlanders and native highlanders: relationships with oxidative stress and role of sympathoexcitation. J Physiol. 2014 Mar 1;592(Pt 5):1009-24.

The residents of the Himalayan valleys belonging to the Sherpa ethnicity are a unique example of a population adapted to high altitudes but not only [21-23]. Life according to traditional lifestyle in a hostile environment, far away from the city centers, in absence of road infrastructure and motorvehicles implicate high-energy expenditure for movement and transportation. In addition to this, the occupational activity based principally on agriculture and yard activity performed by traditional means without industrialized machines exposes the subjects to a heavy workload. For these reasons, the population living in these environment is chronically exposed to high-intensity occupational PA and thus represents a unique example to study its effects on CV health.

Biomarkers of vascular subclinical damage are potentially useful tools for assessment of CV risk beyond theclassical risk factors [24]. Intima-media thickness (IMT) of the common carotid artery and carotid-femoral pulse wave velocity (PWV) are well-known biomarker of atherosclerosis associated with the development of CV diseases [25-28] commonly used as surrogate endpoints in various clinical trials [29]. Flow mediated dilation (FMD) of the brachial artery, although not being considered a surrogate endpoint [29], is a marker of endothelial dysfunction predictive of major CV events, widely used in clinical research [30-33].

The aim of this study was to investigate the association between occupational PA and subclinical vascular damage among a moderate altitude-population exposed to high levels of occupational PA. In order to identify the presence of vascular damage we measured a panel of markers of subclinical atherosclerosis and vascular dysfunction such as FMD, carotid and aortic stiffness and carotid IMT.

\section{Methods}

\section{Study population}

Seventy-two high altitude subjects belonging to the Sherpa ethnicity were enrolled, currently living in the rural village of Chauricharka, Nepal, chronically exposed to high-intensity occupational PA. Inclusion criteria were: age between 16 and 75 years old, residential status in the village, apparent 
good health status and written informed consent. Exclusion criteria were: alcohol use, active neoplasm, infective diseases, pregnancy and the absence of informed consent. All the subjects enrolled were aware of the purposes of the study and gave written informed consent. The study was approved by the Nepal Health Research Council (NHRC) and the Nepal Academy of Science and Technology (NAST), Kathmandu, Nepal and registered (Clinical Trials Gov Registration \# NCT01329159).

\section{Experimental protocol}

All measurements were performed in the morning, in a quiet room. Medical history was taken by Nepalese physicians with particular attention to the assessment of cardiovascular risk factors. Brachial BP was measured in supine position after at least 10 minutes of rest. Three measurements at 2-minutes intervals were taken, and averaged on the last two measurements. Finger $\mathrm{O}_{2}$ saturation, weight, height and waist circumference were also taken and BMI was calculated. Blood samples were taken from 55 patients for laboratory tests. Lipid profile, electrolytes, blood glucose, hemoglobin and renal function were all determined according to the standard laboratory procedures in order to discriminate between the role of occupational PA and the role of other cardiovascular risk factors. Framingham risk score (FRS) was also calculated.

\section{Assessment of physical activity}

PA levels were assessed using the international physical activity questionnaire (IPAQ). IPAQ is a standardized, validated questionnaire used as an international measure for PA [34]. The questionnaire used in the present study was the long interview-administered version collecting information about 4 different types of PA: household and yard activity (6 items), work activity (7 items), transportation activity (6 items), and leisure time PA (6 items). Two more questions assess the time spent sitting to indicate a sedentary behavior. All subjects were interviewed and the questionnaire was administered. The data collected were reported as a continuous measure in METminutes/week. MET values were calculated for the above mentioned types of PA and the total physical activity score (TPAS) was extracted according to the guidelines for data processing and analyzing of IPAQ [35]. Physical activity levels were then classified into 3 levels: low, moderate and high-intensity.

\section{Carotid ultrasound assessment}

Common carotid artery scans were obtained in all subjects by high-resolution ultrasound with a 10 MHz linear probe (MyLab25, ESAOTE, Florence, Italy). In a segment free of atherosclerotic plaques, two 10-second clips were recorded from each common carotid artery $(1 \mathrm{~cm}$ proximal to the carotid bulb in a region $1 \mathrm{~cm}$-wide) and analyzed offline by Carotid Studio (Cardiovascular Suite, Quipu srl, Pisa, Italy), a software for the automatic evaluation of carotid diameter, IMT and stiffness [36]. The following indices were calculated: Carotid distension $(\Delta \mathrm{D})$, that is the stroke change in diameter, calculated as the difference between the systolic and diastolic diameter values; Cross-sectional distensibility coefficient (DC), calculated as $\mathrm{DC}=\Delta \mathrm{A} /(\mathrm{A}$ *carotid PP), where $\mathrm{A}$ is the diastolic lumen area, and $\Delta \mathrm{A}$ is the stroke change in lumen area; Carotid stiffness (CS), calculated using the Moens-Korteweg equation, $\left(\rho^{*} \mathrm{DC}\right)^{-1 / 2}$, where $\rho$ is the blood density. Carotid lumen area was calculated from the diameter values, assuming the cross-section of the artery as circular. C-IMT was automatically measured on the same image sequences as the mean of the 
relative values of 10 seconds. Parameters were indicated as mean of the left and right common carotid artery.

\section{Carotid-femoral pulse wave velocity}

Carotid-femoral pulse wave velocity (PWV) were measured by applanation tonometry (Sphygmocor CPVH, Atcor Medical, Australia), according to the international recommendations [37]. Waveforms were recorded at the two recording sites, sequentially. PWV was calculated as the ratio between the subtracted distance between the two recording sites [(femoral-suprasternal distance) - (carotid-suprasternal distance)] and wave transit time. $P W V=$ distance/transit time. Wave transit time was estimated by the foot-to-method. Two consecutive measurements were taken and then averaged.

\section{Endothelium dependent dilation of the brachial artery}

All measurements were taken by the same operator (R.M.B.) with the same equipment and technique. Brachial artery scans were taken by a high resolution ultrasound with a $10 \mathrm{MHz}$ linear probe (MyLab25; ESAOTE, Florence, Italy). All patients were studied at rest in a temperaturecontrolled room at least $4 \mathrm{~h}$ after meal. Patients were instructed to lie quietly in a supine position for 10 min before the study. Endothelium-dependent function was assessed by FMD as increase of the brachial artery diameter in response to increased blood flow [37]. A B-mode scan of the left brachial artery was taken in longitudinal section 5-10 cm above with the probe being fixed by a stereotactic clamp to ensure a stable recording. After a 1-minute baseline recording, a pediatric cuff was inflated around the forearm just below the elbow, for 5 minutes at $300 \mathrm{~mm} \mathrm{Hg}$, and then deflated to induce reactive hyperemia. The scans were analyzed by a real time computerized detection system which allows a continuous, simultaneous measurement of both flow velocity (FV) and brachial artery diameter (Cardiovascular Suite, Quipu srl; Pisa, Italy). Endotheliumindependent dilation was assessed as the dilation induced by sublingual administration of $25 \mu \mathrm{g}$ of glyceril trinitrate (GNT). FMD and GNT response were calculated as the percentage increase in brachial artery diameter above baseline.

\section{Statistical analysis}

SPSS version 21.0 (IBM Corp., Armonk, NY, USA) software were employed for statistical analysis. Clinical, vascular and PA characteristics of the sample were assessed using descriptive statistics: continuous variables were expressed as median, mean \& standard deviation and categorical variables as counts and percentages. Pearson's rho correlation analysis was carried out in order to compare markers of vascular damage and different types of PA. Multiple regression models were performed to evaluate the relation between PWV, carotid distension, CC and CS (as dependent variables) and age, sex, oxygen saturation, mean blood pressure, LDL and occupational PA score (as independent variables). Before multiple regression model analysis, covariates were individually tested with a univariate linear regression model.

\section{Results}

In the population 72 subjects were studied. Our population was a relatively young population, mean age $45 \pm 15$, men 23 (31.9\%). Results are shown in table 1 . As expected, oxygen saturation and mean hemoglobin levels are compatible with a good acclimatization to moderate altitudes, typical of this population. The prevalence of hypertension was 33.3\% while hypercholesterolemia and low HDL 
were present in $44 \%$ and $49 \%$, the prevalence of diabetes, smoke, obesity and chronic kidney disease was low as shown in table 1 . FRS was calculated for 48 (67\%) subjects and median value was $1 \%$.

\begin{tabular}{ll}
\hline Clinical characteristics & Mean \pm SD \\
\hline Age & $42 \pm 15$ \\
Men (\%) & 31.9 \\
Weight & $61.77 \pm 10.45$ \\
Height & $158 \pm 8$ \\
BMI & $24.6 \pm 4.2$ \\
Waist circumference & $86.7 \pm 9.8$ \\
Hypertension (\%) & 33.3 \\
Diabetes (\%) & 2.8 \\
\hline Smoke (\%) & 6.9 \\
\hline Obesity (\%) & 9.7 \\
Hypercholesterolemy (\%) & 44 \\
Low HDL (\%) & 49 \\
Chronic kidney disease (\%) & 7.3 \\
HR (bpm) & $68 \pm 10$ \\
SBP (mmHg) & $129.4 \pm 16.5$ \\
DBP (mmHg) & $79.2 \pm 10.6$ \\
PP(mmHg) & $50.2 \pm 9.6$ \\
\hline Oxygen saturation (\%) & $94.1 \pm 1.6$ \\
\hline LDL (mg/dl) & $138.4 \pm 31.2$ \\
Total Choleterol (mg/dl) & $207.7 \pm 45.6$ \\
\hline HDL (mg/dl) & $50.7 \pm 13.5$ \\
\hline Tryglicerides (mg/dl) & $129.4 \pm 72.3$ \\
\hline Hemoglobin (mg/dl & $15.0 \pm 1.3$ \\
Glucose (mg/dl) & $82.7 \pm 20.7$ \\
\hline Creatinine (mg/dl) & $0.86 \pm 0.27$ \\
GFR (60ml/min1.73mq) & $91.6 \pm 24.2$ \\
Framingham risk score (\%) & $1(<1 ;>3)$ \\
\hline
\end{tabular}

Table 1: Clinical characteristics of the study population. BMI: body mass index; HR: heart rate; SBP: systolic blood pressure; DBP: diastolic blood pressure; PP: pulse pressure;

The vascular study was feasible in the subjects and PWV, carotid ultrasound and FMD were successfully assessed in 63 (87.5\%), 69 (95.8\%) and 71 (98.6\%) subjects respectively. Mean values are shown in table 2. Mean PWV was $7.2 \pm 1.6 \mathrm{~m} / \mathrm{s}$ while IMT was $0.55 \pm 0.14 \mathrm{~mm}$. The endothelial function evaluated by FMD and GTN were normal $(4.13 \pm 2.34 \%$ and $7.3 \pm 3.0 \%$ respectively).

\begin{tabular}{ll}
\hline Vascular characteristics & Mean \pm SD \\
\hline PWV (m/s) & $7.2 \pm 1.6$ \\
Carotid IMT (mm) & $0.55 \pm 0.14$ \\
Mean carotid diameter (mm) & $7.48 \pm 0.96$ \\
Carotid distension $(\mathrm{mm})$ & $0.55 \pm 0.15$ \\
CC & $0.99 \pm 0.33$ \\
DC & $28.35 \pm 24.72$ \\
CS (m/s) & $6.65 \pm 1.40$ \\
BA baseline diameter $(\mathrm{mm})$ & $4.16 \pm 0.62$ \\
FMD (\%) & $4.13 \pm 2.34$ \\
Baseline SR & $167.6 \pm 82.5$ \\
Maximum SR & $923.1 \pm 446.5$ \\
SR AUC & $30746.7 \pm 18836.6$ \\
GTN (\%) & $7.3 \pm 3.0$ \\
\hline
\end{tabular}

Table 2: Vascular characteristics of the study population. PWV: pulse wave velocity; IMT: intima-media thickness; CC: compliance coefficient; DC; distensibility coefficient; CS: carotid 
stiffness; BA: brachial artery; FMD: flow mediated dilation; SR: shear rate; SR AUC; shear rate area under the curve; GTN: glyceril trinitrate mediated dilation.

IPAQ was successfully administered to all the subjects and PA scores were calculated for all 4 types of PA. Work and yard activity were included under the same category as occupational PA, while transportation and leisure time PA were taken into account separately. Results are shown in table 3. TPAS was $12806 \pm 5443$ MET-minutes/week, with $97 \%$ of the population performing a high level of PA. Occupational activity was the principal form of PA being responsible for approximately $77 \%$ of total energy expenditure among the inhabitants. Mean MET-minutes values for occupational PA was $9860 \pm 5385$ MET-minutes/week while leisure time PA score was $765 \pm 1423$ MET-minutes/week and represents only $6 \%$ of TPAS. Mean average sitting time was $184 \pm 91$ minutes/day.

\begin{tabular}{lllll}
\hline & Mean & Mean \% & Median (25-75\%) & Median \% \\
\hline Trasportation PA (MET-minutes/week) & $2180 \pm 2452$ & $17 \%$ & $1386(0-2772)$ & $11,2 \%$ \\
Leisure-time PA (MET-minutes/week) & $765 \pm 1423$ & $6 \%$ & $0(0-1440)$ & $0,0 \%$ \\
Occupational PA (MET-minutes/week) & $9860 \pm 5385$ & $77 \%$ & $10080(6720-13440)$ & $80,9 \%$ \\
TPAS (MET-minutes/week) & $12806 \pm 5443$ & & $12558(9702-15120)$ & $180(120-240)$ \\
Average sitting time (min) & $184 \pm 91$ & & & \\
\hline
\end{tabular}

Table 3. Physical activity (PA) levels of the study population (IPAQ). TPAS: total physical activity score.

PWV and CS showed a significant positive correlation with both occupational PA score and TPAS while carotid distension and carotid CC presented an inverse correlation (Table 4). No significant association was found with leisure time PA, transportation/movement activity or total sitting time.

IMT, carotid diameter, carotid DC, FMD, baseline SR, SR max, SR area, SR area to max, GTN were not significantly associated with neither occupational PA score nor TPAS. However, SR max, SR area and SR area to max were found to be significantly correlated with leisure time PA score while carotid DC was associated with transportation activity (appendix 1).

\begin{tabular}{|c|c|c|c|c|c|c|}
\hline & & $\begin{array}{l}\text { OCCUPATIONAL } \\
\text { ACTIVITY }\end{array}$ & $\begin{array}{l}\text { TRANSPORTATION } \\
\text { ACTIVITY }\end{array}$ & $\begin{array}{l}\text { LEISURE TIME } \\
\text { PHYSICAL } \\
\text { ACTIVITY }\end{array}$ & $\begin{array}{l}\text { TOTAL } \\
\text { PHYSICAL } \\
\text { ACTIVITY } \\
\text { SCORE }\end{array}$ & $\begin{array}{l}\text { TOTAL } \\
\text { SITTING } \\
\text { TIME }\end{array}$ \\
\hline PWV & $\begin{array}{l}\text { Pearson } \\
\text { correlation }\end{array}$ & 0,474 & 0,133 & $-0,102$ & 0,503 & $-0,045$ \\
\hline$n=62$ & $\mathrm{p}$ & $0,001^{*}$ & 0,302 & 0,430 & $0,001 *$ & 0,726 \\
\hline $\begin{array}{l}\text { Carotid } \\
\text { distension }\end{array}$ & $\begin{array}{l}\text { Pearson } \\
\text { correlation }\end{array}$ &,- 320 & $-0,014$ & $-0,114$ &,- 346 & 0,184 \\
\hline$n=65$ & $\mathrm{p}$ & 0,009* & 0,915 & 0,366 & $0,005^{*}$ & 0,143 \\
\hline CC & $\begin{array}{l}\text { Pearson } \\
\text { correlation }\end{array}$ &,- 302 & $-0,056$ & $-0,056$ &,- 334 & 0,057 \\
\hline$n=65$ & $\mathrm{p}$ & $0,015^{*}$ & 0,660 & 0,656 & 0,007* & 0,651 \\
\hline CS & $\begin{array}{l}\text { Pearson } \\
\text { correlation }\end{array}$ & 0,289 & $-0,083$ & 0,157 & 0,283 & $-0,017$ \\
\hline$n=65$ & $\mathrm{p}$ & 0,019* & 0,509 & 0,213 & 0,022* & 0,890 \\
\hline
\end{tabular}


Table 4. Spearman correlation between markers of vascular damage and physical activity. PWV: pulse wave velocity; CC: compliance coefficient; DC; distensibility coefficient; CS: carotid stiffness;

At the univariate analysis PWV showed a significant positive association with occupational PA score $(\mathrm{R}=0.474 ; \mathrm{p}<0.001)$ and TPAS (Figure 1$)$ while no association was found neither with leisure time PA nor transportation activity. In the multiple regression analysis including age, sex, mean blood pressure, oxygen saturation, blood LDL concentration and occupational PA score only age ( $\beta$ $=0.439 ; \mathrm{p}=0.002)$, oxygen saturation $(\beta=0.309 ; \mathrm{p}=0.014)$ and occupational PA $(\beta=0.403 ; \mathrm{p}=0.001)$ remained independent predictors of PWV (full model R=0.718) (Table 5).

Similar results were found also for carotid stiffness indexes. At the univariate analysis carotid distension and CC were found to be inversely correlated with occupational PA levels $(\mathrm{R}=-0.32$, $\mathrm{p}=0.09 ; \mathrm{R}=-0.30, \mathrm{p}=0.015)$ while $\mathrm{CS}$ was found to be positively correlated $(\mathrm{R}=0.29, \mathrm{p}=0.019)$. However, in the multivariate model, including age, sex, mean arterial pressure, oxygen saturation, blood LDL concentration and occupational PA score significance was lost and only age and mean arterial pressure remained independent predictors of CS (table 5).

In the univariate regression model, FRS showed a small yet significant association with PWV ( $\beta=0.324, p=0.039$; full model $\mathrm{R}=0.324$ ). In a multiple regression model including occupational $\mathrm{PA}$ and FRS as possible predictors of PWV, occupational physical activity was not only significantly associated with PWV $(\beta=0.535, p=0.00009)$ but also increased the association between FRS and PWV ( $\beta=0.397, p=0.002$; full model $\mathrm{R}=0.672$ ) (Table 5).

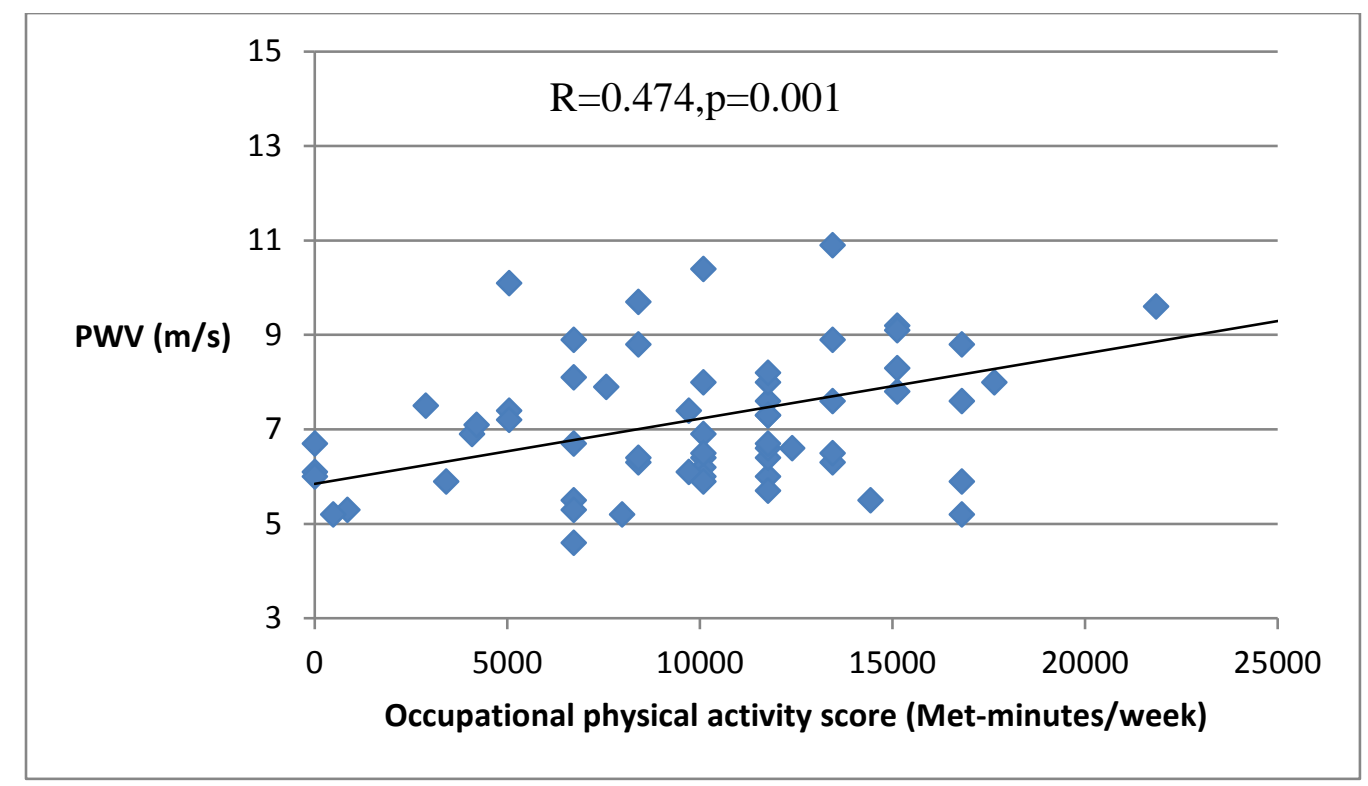

Figure 1. Spearman correlation between pulse wave velocity (PWV) and occupational physical activity score

\begin{tabular}{|l|l|l|l|}
\hline & \multicolumn{1}{|c|}{ Variable } & $\boldsymbol{\beta}$ & P value \\
\hline \multirow{2}{*}{$\begin{array}{l}\text { Variable PWV } \\
\text { Full R=0.718 }\end{array}$} & Age & 0.439 & $\mathbf{0 . 0 0 2}$ \\
\cline { 2 - 4 } & Sex & 0.042 & 0.70 \\
\cline { 2 - 4 } & SO2 & 0.309 & 0.014 \\
\hline
\end{tabular}




\begin{tabular}{|l|l|l|l|}
\hline \multirow{4}{*}{$\begin{array}{l}\text { Variable CS } \\
\text { Full R=0.757 }\end{array}$} & Mean blood pressure & 0.182 & 0.11 \\
\cline { 2 - 4 } & Occupational physical activity score & 0.403 & $\mathbf{0 . 0 0 1}$ \\
\cline { 2 - 4 } & LDL & 0.100 & 0.5 \\
\cline { 2 - 4 } & Sex & 0.512 & $\mathbf{0 . 0 0 0 2}$ \\
\cline { 2 - 4 } & Oxygen saturation & -0.103 & 0.32 \\
\cline { 2 - 4 } & Mean blood pressure & -0.132 & 0.24 \\
\cline { 2 - 4 } & Occupational physical activity score & 0.028 & 0.80 \\
\cline { 2 - 4 } & LDL & 0.001 & 0.99 \\
\hline Variable PWV & Framingham risk score & 0.324 & $\mathbf{0 . 0 3 9}$ \\
Full R=0.324 & & 0.303 & $\mathbf{0 . 0 1}$ \\
\hline $\begin{array}{l}\text { Variable PVV } \\
\text { Full R=0.672 }\end{array}$ & Framingham risk score & 0.002 \\
\cline { 2 - 4 } & Occupational physical activity score & 0.535 & 0.00009 \\
\hline
\end{tabular}

Table 5. Multivariate correlation models exploring determinants of arterial stiffness

In the univariate regression model, FRS showed a small yet significant association with PWV ( $\beta=0.324$, $p=0.039$; full model $R=0.324$ ). In a multiple regression model including occupational physical activity and FRS as possible predictors of PWV, occupational physical activity was not only significantly associated with PWV $(\beta=0.535, \mathrm{p}=0.00009)$ but also increased the association between FRS and PWV ( $\beta=0.397, p=0.002$; full model $\mathrm{R}=0.672$ ) (Table 5)

\section{Discussion}

The aim of this study was to investigate the association between occupational PA and subclinical vascular damage. Our study population was a relatively young population, born and resident at moderate-altitude.. Among the traditional CV risk factors we found increased prevalence of hypertension, increased concentration of LDL and reduced concentration of HDL which indicates an exposure to the traditional risk among this population. The increased prevalence of these conditions could be attributed to the dietary habits characterized by an increased use of salt and lipid-contain products. Moreover, the reduced availability of drugs, mainly due to the geographic location of the village far away from the inhabited centers and the low socio-economic status, makes it difficult to treat these conditions. However, FRS was found to be low with a median 10years risk of $\mathrm{CV}$ events of $1 \%$. The low FRS found is due principally to the young age of the population.

Regarding the vascular structure, PWV, IMT were not pathological [38, 39] indicating a reduced arterial atherosclerotic damage. On the other hand, FMD and GTN, although there are no reference values (referenza RM), were found to be relatively reduced indicating a possible endothelial dysfunction in the subjects under investigation. In fact, our group, in a previous study found a significantly reduced FMD among the Sherpa population compared to Caucasians at sea level indicating an endothelial dysfunction among this population [24]. The reduced FMD dilation may be also a consequence of the high level of physical activity performed by this population. Although there are not similar studies that confirm the association between reduced FMD and occupational PA, an association with strenuous leisure-time PA has already been reported (referenza RM). ?

The subjects enrolled in this study were chronically exposed to strenuous PA level. However in contrast with most of the studies were leisure time PA represents the principal form of PA, in our study population it was the less represented. In fact, occupational PA, principally based on agriculture and yard activity, was the principal form of PA, being responsible for approximately $80 \%$ of total daily energy expenditure. Occupational PA was found to be significantly correlated 
with different markers of arterial stiffness while no correlation was found with carotid IMT or endothelial function. In particular occupational PA significantly correlates with increased PWV and carotid stiffness and with reduced carotid distension and CC suggesting an increase in both aortic and carotid stiffness among more active subjects. The association with PWV remained significant even after adjusting for age, sex, oxygen saturation, mean blood pressure and LDL concentration while significance was lost for carotid stiffness and distensibility. Occupational PA showed to be significantly correlated with PWV even after adjusting for FRS remaining an independent determinant of aortic stiffness. In the multiple regression analysis the association of occupational PA and FRS was found to be better predictors of PWV than FRS alone. No significant correlation was found between leisure time PA and markers of arterial stiffness or function.

Although there are not studies that confirm the association between occupational PA and PWV, many studies have found an increased risk of CV diseases among people exposed to high levels of occupational PA [14-17, 19, 40-42]. In the present study we found a significant positive correlation, independently from other CV risk factors, between occupational PA and PWV. By taking into account the fact that PWV is well known biomarker of subclinical vascular damage predictive of CV events, widely used as a surrogate endpoint of atherosclerosis [27-29], our findings suggest an association between high intensity occupational physical activity and overall CV. Our result is partly confirmed by Krause et.al, who showed that high intensity occupational PA is associated with an accelerated progression of carotid atherosclerosis [18, 43], measured as carotid IMT. Although IMT and PWV are 2 different markers of vascular damage, they both reflect the progression of atherosclerosis and both correlate with CV risk [29]. The present study also shows how the assessment of occupational PA increases the association between FRS and PWV and thus should be evaluated during CV risk assessment.

\section{Physiopatogical mechanisms}

The physiopatological mechanisms by which high-intensity occupational physical activity increases the risk of CV diseases are not completely clear. According to the hemodynamic theory of the atherosclerosis [44], PA triggers hemodynamic turbulences and increases endothelial shear stress by increasing the heart rate and consequently reducing the time spent in systole when wall shear stress is optimal and increasing the time spent in diastole when shear stress is suboptimal[18, 44, 45]. The increased shear stress and blood turbulences may then induce endothelial damage which causes the absorption of lipids into the arterial intima layer triggering the atherosclerotic process, which is responsible for increased arterial stiffness and leads to the formation of atherosclerotic plaques [46].

This mechanism is the same for high intensity leisure physical activity. I think we should discuss also this point.

Another point to take into account is the oxygen desaturation that likely occurs even in acclimatized subjects during high intensity physical activity. We have not this data but we know, for example, that in OSAS and COPD subjects, endothelial dysfunction is related to more severe disease and the presence of oxygen desaturation during the 6MWT (i.e. Cinarka $\mathrm{H}$ et al. Arterial stiffness measured via carotid femoral pulse wave velocity is associated with disease severity in COPD. Respir Care. $2014 \mathrm{Feb} ; 59(2): 274-80$.

\section{Limitations}


Some limitations of our study must be acknowledged. Dietary habits of this population or moderate altitude exposure could modify aortic stiffness and distensibilty. Although Parati et. al. showed that PWV does not change after exposure to high altitude [47], the lack of differentiation between the effect of PA and these confounders could be a limit of our study. Moreover the absence of a follow up study to better assess the association between physical activity and future CV events could be another limit. A follow up study in this population is going to be scheduled in the next 2 years.

\section{Conclusions}

In conclusion, the high-intensity occupational physical activity shows a positive, independent association with arterial stiffness in Himalayan high altitude dwellers. This study suggests how occupational PA may be a new CV risk factor and thus should be included in the assessment of overall CV risk. Prospective follow up studies are needed to better understand the association and its correlation with future CV events.

Acknowledgments: This work was conducted in the framework of SHARE - Stations at High Altitude for Research on the Environment Project, promoted by Ev-K2-CNR. Founded by Ev-K2-CNR (Italian National Research Council). 
1. Go, A.S., et al., Heart disease and stroke statistics--2014 update: a report from the American Heart Association. Circulation. 129(3): p. e28-e292.

2. Daniels, S.R., Prevention of atherosclerotic cardiovascular disease: what is the best approach and how early should we start? J Am Coll Cardiol, 2014. 63(25 Pt A): p. 2786-8.

3. Alagona, P., Jr. and T.A. Ahmad, Cardiovascular disease risk assessment and prevention: current guidelines and limitations. Med Clin North Am. 99(4): p. 711-31.

4. Van Camp, G., Cardiovascular disease prevention. Acta Clin Belg. 69(6): p. 407-11.

5. Lavie, C.J., et al., Exercise and the cardiovascular system: clinical science and cardiovascular outcomes. Circ Res, 2015. 117(2): p. 207-19.

6. Yusuf, S., et al., Effect of potentially modifiable risk factors associated with myocardial infarction in 52 countries (the INTERHEART study): case-control study. Lancet, 2004. 364(9438): p. 937-52.

7. Luke, A., et al., Assessing physical activity and its relationship to cardiovascular risk factors: NHANES 2003-2006. BMC Public Health. 11: p. 387.

8. Fitzgerald, J.D., et al., Association of objectively measured physical activity with cardiovascular risk in mobility-limited older adults. J Am Heart Assoc. 4(2).

9. Leon-Latre, M., et al., Sedentary lifestyle and its relation to cardiovascular risk factors, insulin resistance and inflammatory profile. Rev Esp Cardiol (Engl Ed), 2014. 67(6): p. 449-55.

10. Mac Ananey, O., et al., Inverse Relationship Between Physical Activity, Adiposity and Arterial Stiffness in Healthy Middle-aged Subjects. J Phys Act Health, 2015.

11. Physical Activity Guidelines Advisory Committee report, 2008. To the Secretary of Health and Human Services. Part A: executive summary. Nutr Rev, 2009. 67(2): p. 114-20.

12. Wang, Y., et al., Occupational, commuting, and leisure-time physical activity in relation to heart failure among finnish men and women. J Am Coll Cardiol. 56(14): p. 1140-8.

13. Salonen, J.T., P. Puska, and J. Tuomilehto, Physical activity and risk of myocardial infarction, cerebral stroke and death: a longitudinal study in Eastern Finland. Am J Epidemiol, 1982. 115(4): p. 526-37.

14. Stender, M., et al., Physical activity at work and cardiovascular disease risk: results from the MONICA Augsburg study. Int J Epidemiol, 1993. 22(4): p. 644-50.

15. Kristal-Boneh, E., et al., Association of physical activity at work with mortality in Israeli industrial employees: the CORDIS study. J Occup Environ Med, 2000. 42(2): p. 127-35.

16. Raum, E., et al., Heavy physical activity: risk or protective factor for cardiovascular disease? A life course perspective. Ann Epidemiol, 2007. 17(6): p. 417-24.

17. Virkkunen, H., et al., Shift work, occupational noise and physical workload with ensuing development of blood pressure and their joint effect on the risk of coronary heart disease. Scand J Work Environ Health, 2007. 33(6): p. 425-34.

18. Krause, N., et al., Occupational physical activity, energy expenditure and 11-year progression of carotid atherosclerosis. Scand J Work Environ Health, 2007. 33(6): p. 405-24.

19. Holtermann, A., et al., Physical demands at work, physical fitness, and 30-year ischaemic heart disease and all-cause mortality in the Copenhagen Male Study. Scand J Work Environ Health, 2010. 36(5): p. 357-65.

20. Richard, A., et al., Effects of leisure-time and occupational physical activity on total mortality risk in NHANES III according to sex, ethnicity, central obesity, and age. J Phys Act Health, 2015. 12(2): p. 184-92.

21. Beall, C.M., Two routes to functional adaptation: Tibetan and Andean high-altitude natives. Proc Natl Acad Sci U S A, 2007. 104 Suppl 1: p. 8655-60.

22. Chen, Q.H., et al., Exercise performance of Tibetan and Han adolescents at altitudes of 3,417 and 4,300 m. J Appl Physiol (1985), 1997. 83(2): p. 661-7.

23. Bruno, R.M., et al., Cardiovascular function in healthy Himalayan high-altitude dwellers. Atherosclerosis, 2014. 236(1): p. 47-53.

24. Bruno, R.M., et al., Intima media thickness, pulse wave velocity, and flow mediated dilation. Cardiovascular ultrasound, 2014. 12: p. 34. 
25. Chambless, L.E., et al., Association of coronary heart disease incidence with carotid arterial wall thickness and major risk factors: the Atherosclerosis Risk in Communities (ARIC) Study, 1987-1993. Am J Epidemiol, 1997. 146(6): p. 483-94.

26. Chambless, L.E., et al., Carotid wall thickness is predictive of incident clinical stroke: the Atherosclerosis Risk in Communities (ARIC) study. Am J Epidemiol, 2000. 151(5): p. 478-87.

27. Laurent, S., et al., Expert consensus document on arterial stiffness: methodological issues and clinical applications. Eur Heart J, 2006. 27(21): p. 2588-605.

28. Vlachopoulos, C., et al., Prediction of cardiovascular events and all-cause mortality with central haemodynamics: a systematic review and meta-analysis. Eur Heart J, 2010. 31(15): p. 1865-71.

29. Vlachopoulos, C., et al., The role of vascular biomarkers for primary and secondary prevention. $A$ position paper from the European Society of Cardiology Working Group on peripheral circulation: Endorsed by the Association for Research into Arterial Structure and Physiology (ARTERY) Society. Atherosclerosis, 2015. 241(2): p. 507-32.

30. Celermajer, D.S., et al., Non-invasive detection of endothelial dysfunction in children and adults at risk of atherosclerosis. Lancet, 1992. 340(8828): p. 1111-5.

31. Flammer, A.J., et al., The assessment of endothelial function: from research into clinical practice. Circulation, 2012. 126(6): p. 753-67.

32. Yeboah, J., et al., Predictive value of brachial flow-mediated dilation for incident cardiovascular events in a population-based study: the multi-ethnic study of atherosclerosis. Circulation, 2009. 120(6): p. 502-9.

33. Bianchini, E., et al., Functional and structural alterations of large arteries: methodological issues. Curr Pharm Des, 2013. 19(13): p. 2390-400.

34. Craig, C.L., et al., International physical activity questionnaire: 12-country reliability and validity. Med Sci Sports Exerc, 2003. 35(8): p. 1381-95.

35. Guidelines for the data processing and analysis of the International Physical Activity Questionnaire

2005.

36. Giannarelli, C., et al., Local carotid stiffness and intima-media thickness assessment by a novel ultrasound-based system in essential hypertension. Atherosclerosis, 2012. 223(2): p. 372-7.

37. Bruno, R.M., et al., Type 2 diabetes mellitus worsens arterial stiffness in hypertensive patients through endothelial dysfunction. Diabetologia, 2012. 55(6): p. 1847-55.

38. Engelen, L., et al., Reference intervals for common carotid intima-media thickness measured with echotracking: relation with risk factors. Eur Heart J, 2013. 34(30): p. 2368-80.

39. Determinants of pulse wave velocity in healthy people and in the presence of cardiovascular risk factors: 'establishing normal and reference values'. Eur Heart J, 2010. 31(19): p. 2338-50.

40. Holtermann, A., et al., The interplay between physical activity at work and during leisure time--risk of ischemic heart disease and all-cause mortality in middle-aged Caucasian men. Scand J Work Environ Health, 2009. 35(6): p. 466-74.

41. Holtermann, A., et al., Fitness, work, and leisure-time physical activity and ischaemic heart disease and all-cause mortality among men with pre-existing cardiovascular disease. Scand J Work Environ Health, 2010. 36(5): p. 366-72.

42. Holtermann, A., et al., Occupational and leisure time physical activity: risk of all-cause mortality and myocardial infarction in the Copenhagen City Heart Study. A prospective cohort study. BMJ Open, 2012. 2(1): p. e000556.

43. Krause, N., et al., Standing at work and progression of carotid atherosclerosis. Scand J Work Environ Health, 2000. 26(3): p. 227-36.

44. Glagov, S., et al., Hemodynamics and atherosclerosis. Insights and perspectives gained from studies of human arteries. Arch Pathol Lab Med, 1988. 112(10): p. 1018-31.

45. Ku, D.N., et al., Pulsatile flow and atherosclerosis in the human carotid bifurcation. Positive correlation between plaque location and low oscillating shear stress. Arteriosclerosis, 1985. 5(3): p. 293-302.

46. Schoen FJ, Cotran RS. Blood vessels. In: Cotran RS, Kumar V, Collins T, Robbins SL, editors. Pathologic basis of disease. Philadelphia (PA): WB Saunders Company; 1999. 
47. Parati, G., et al., Effects of acetazolamide on central blood pressure, peripheral blood pressure, and arterial distensibility at acute high altitude exposure. Eur Heart J, 2013. 34(10): p. 759-66. 